\title{
Helicobacter pylori infection and esophageal adenocarcinoma: a review and a personal view
}

\author{
Stergios A. Polyzos, Christos Zeglinas, Fotini Artemaki, Michael Doulberis, Evangelos Kazakos, \\ Panagiotis Katsinelos, Jannis Kountouras
}

Aristotle University of Thessaloniki, Ippokration Hospital, Thessaloniki, Macedonia, Greece

\section{Abstract}

\begin{abstract}
Esophageal adenocarcinoma (EAC) is etiologically associated with gastroesophageal reflux disease (GERD). There is evidence to support the sequence GERD, Barrett's esophagus (BE), dysplasia, and finally EAC, with Helicobacter pylori (H. pylori) being implicated in each step to EAC. On the other side of this relation stands the hypothesis of the protective role of $H$. pylori against EAC. Based on this controversy, our aim was to review the literature, specifically original clinical studies and metaanalyses linking $H$. pylori infection with EAC, but also to provide our personal and others' relative views on this topic. From a total of 827 articles retrieved, 10 original clinical studies and 6 metaanalyses met the inclusion criteria. Original studies provided inconclusive data on an inverse or a neutral association between $H$. pylori infection and EAC, whereas meta-analyses of observational studies favor an inverse association. Despite these data, we consider that the positive association between $H$. pylori infection and GERD or BE, but not EAC, is seemingly a paradox. Likewise, the oncogenic effect of $H$. pylori infection on gastric and colon cancer, but not on EAC, also seems to be a paradox. In this regard, well-designed prospective cohort studies with a powered sample size are required, in which potential confounders should be taken into consideration since their design.
\end{abstract}

Keywords Helicobacter pylori, esophageal adenocarcinoma, Barrett's esophagus, gastroesophageal reflux disease

Ann Gastroenterol 2018; 31 (1): 1-6

\section{Introduction}

Helicobacter pylori (H. pylori) is a common bacterium and infects almost half of the global population [1], being strongly associated with upper gastrointestinal morbidity. Its prevalence is still high in most countries; there were approximately 4.4 billion individuals with $H$. pylori infection worldwide in 2015, and $H$. pylori remains highly prevalent in certain ethnic populations and in migrants moving from high prevalence countries [1]. The primary pathogenic role of $H$. pylori in peptic ulcer formation is supported by robust evidence [2], and H. pylori was recognized as a true class I carcinogen for gastric cancer by the International Agency for Research on Cancer [3] and the World Health Organization

Department of Medicine, Second Medical Clinic, Aristotle University of Thessaloniki, Ippokration Hospital, Thessaloniki, Macedonia, Greece

Conflict of Interest: None

Correspondence to: Jannis Kountouras, MD, PhD, Gastroenterologist, Professor of Medicine, 8 Fanariou St, Byzantio, 55133 Thessaloniki,

Macedonia, Greece, e-mail: jannis@auth.gr

Received 12 July 2017; accepted 18 October 2017; published online 16 November 2017

DOI: https://doi.org/10.20524/aog.2017.0213 in 1994. On top of this, numerous studies claim to have implicated $H$. pylori in a long list of systemic disorders, including cardio-cerebrovascular [4,5], degenerative [6-8], and metabolic syndrome (MetS)-related conditions $[4,9]$. Likewise, the accumulated oncology literature suggests an etiological relation of $H$. pylori with extra-gastric neoplasms, such as pancreatic [10], colorectal [11-13], and esophageal cancers, at least in some subpopulations [14].

Esophageal cancer is among the most frequent neoplasms, a main cause of cancer-related deaths worldwide and a clinically challenging disease requiring a multidisciplinary approach [15]. Esophageal cancer is divided into two histological types: esophageal squamous cell carcinoma (ESCC), associated mostly with environmental risk factors (e.g., smoking and alcohol consumption), and esophageal adenocarcinoma (EAC) located close to the gastroesophageal junction, etiologically coupled with gastroesophageal reflux disease (GERD). In the westernized population, the incidence of EAC increased sharply, displacing ESCC, the latter accounting for most of the incidence of esophageal cancer 50 years ago $[16,17]$.

Current evidence for the protective or harmful effect of H. pylori on EAC is conflicting. On this basis, we aimed to review the literature, specifically original clinical studies and meta-analyses linking $H$. pylori infection with EAC, but also to provide our personal and others' relative views on this topic. 


\section{Literature review}

\section{Materials and methods}

A literature search was carried out in the PubMed database using the following query, developed from a combination of MeSH and non-MESH terms: ((Helicobacter pylori) OR $(H p)$ OR (H. pylori)) AND ((esophageal neoplasm) OR (esophageal carcinoma) OR (esophageal cancer) OR (esophageal adenocarcinoma)). Additional studies were identified by hand search from references of the eligible articles and commentaries on the current topic ("hand searching"). The search was completed on June 25,2017 . The selection process was performed independently by two researchers ( $\mathrm{CZ}$ and $\mathrm{JK})$.

Eligibility was based on the following inclusion criteria: clinical studies or meta-analyses reporting on the association between $H$. pylori and EAC; and histological confirmation of EAC. Exclusion criteria were: studies in languages other than English, abstracts of conferences; reviews; commentaries; editorials; and experimental studies.

Subsequently, a quality evaluation of the eligible original studies was conducted. For the purposes of the quality assessment, the Methodological Index for Non-Randomized Studies (MINORS) was used. MINORS is a validated and established index for evaluating the methodological quality of non-randomized studies. This index involves 12 criteria, 8 of which have been designed for non-comparative studies, whereas the other 4 criteria apply to comparative studies. These criteria are scored on a scale developed by Slim et al [18]: 0 (not reported), 1 (reported but inadequate), and 2 (reported and adequate). The maximum score for comparative studies is 24 and for the 8 -item index is 16 , while the minimum score is 0 .

The aforementioned two reviewers ( $\mathrm{CZ}$ and $\mathrm{JK}$ ) independently evaluated each study according to the MINORS index and any scoring differences were discussed until consensus was reached. With regard to the 12 -item index, a score greater than 16 was indicative of well-designed studies [19,20]. No threshold is currently proposed for the 8-item index. We aimed to evaluate the randomized controlled trials (RCTs) with the Cochrane tool, but no RCT was retrieved.

\section{Results}

\section{Selection process}

The initial search in PubMed resulted in the retrieval of 607 articles. Through manual searching, 220 articles were added, bringing the total to 827 . After the initial screening on the basis of their title and/or abstract, 784 articles were excluded and the full text of 43 articles was evaluated for eligibility. Finally, 10 original studies and 6 meta-analyses were selected. A flowchart illustrating the selection process is presented in Fig. 1.

No RCTs fulfilling the eligibility criteria were identified. This was not unexpected, since the preferred study design for investigating prognostic and risk factors is the cohort study, followed by the case control study in evidence-based medicine (www.cebm.net/ocebm-levels-of-evidence).

\section{Methodological quality}

The MINORS 8-item index applied to all selected original studies and the results of the MINORS scoring are presented in Table 1. MINORS scores ranged from 3-11. The major limitations on the methodology of the selected studies were a retrospective design and a non-calculated or small sample size. Cohen's kappa coefficient, measuring the inter-rater agreement (CZ and JK) for each MINORS item, ranged between 0.84 and 0.92 (all $\mathrm{P}<0.01)$.

\section{Summary of original studies included}

Among the selected original studies, five reported an inverse relation between $H$. pylori infection and EAC [21-25], whereas the other five reported no association between H. pylori infection and EAC [26-30]. Notably, the studies scored higher suggested a neutral relationship between $H$. pylori and EAC [26,30]. Siman et al observed no association between EAC and $H$. pylori seropositivity, cytotoxin-associated gene A (CagA) seropositivity or CagA seropositivity among $H$. pylori seropositive subjects [26]; however, the study may possibly have been underpowered.

\section{Summary of included meta-analyses}

The first meta-analysis of the association between $H$. pylori infection and EAC was published in 2007 [31]. Another four later meta-analyses of observational studies were retrieved on the same topic [32-35]. All the meta-analyses reported lower rates of EAC in $H$. pylori-positive compared with $H$. pylori-negative individuals. Furthermore, all meta-analyses showed lower

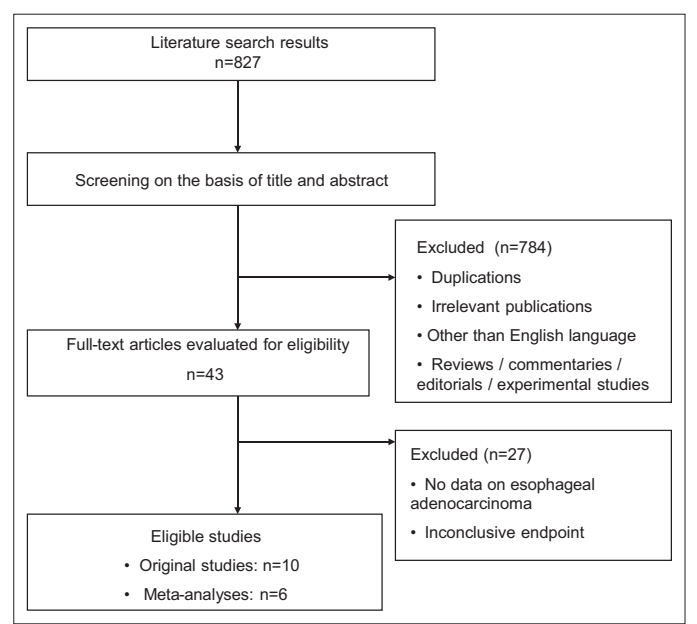

Figure 1 A flowchart presenting the literature search process, according to the Preferred Reporting Items for Systematic reviews and Meta-Analyses (PRISMA) statement 


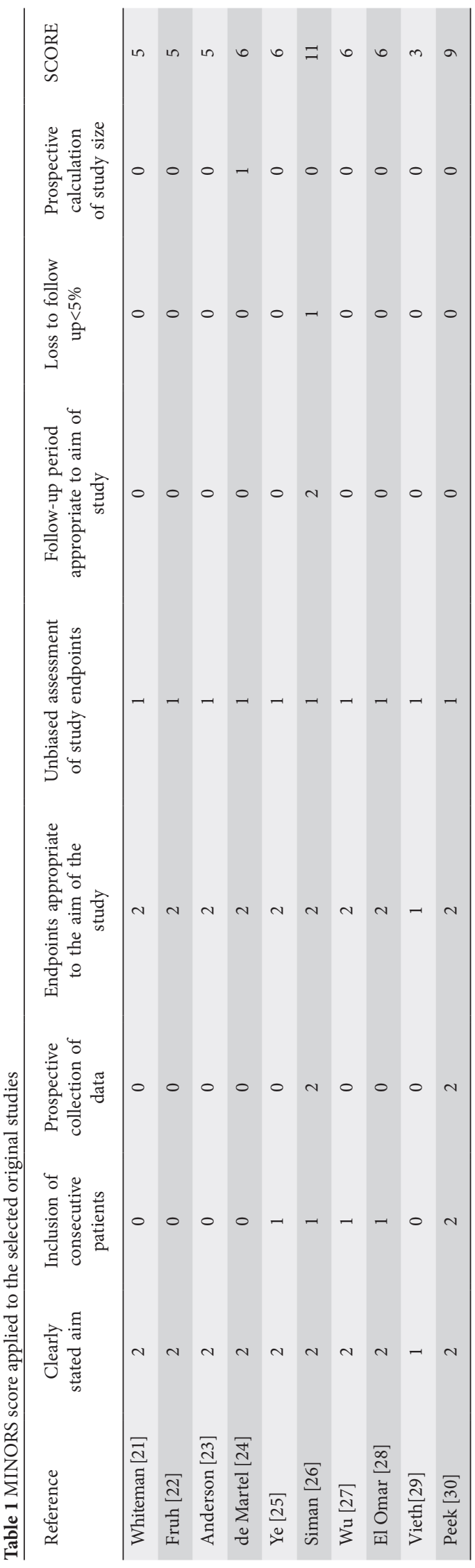

rates of EAC in $H$. pylori CagA-positive individuals compared with controls (H. pylori-negative individuals). H. pylori CagAnegative individuals and controls (H.pylori-negative individuals) had similar EAC rates, as shown in one meta-analysis [33]. As expected, there was overlap of the included studies in all metaanalyses. Although there was heterogeneity in some of the metaanalyses, meta-regression to assess the source of heterogeneity was not performed in any of them.

\section{Personal view}

Although some studies and all meta-analyses reported an inverse association between $H$. pylori infection and EAC, interpreted as a protective effect by some authors, our personal relative consideration and those of others do not agree. Our position starts from a simple question: would a physician propose the potential contamination of $H$. pylori infection in high-risk populations (e.g., obese, cigarette smokers, consumers of high quantities of red or processed meat), so as to protect them from EAC? In our opinion, a randomized controlled trial would never be assigned to answer this question, since it would transgress certain ethical considerations. In this regard, Prof. David Y. Graham maintained that H. pylori is not and never was "protective" against anything [36]. Fig. 2 summarizes the main results of the review, but also the main points of our consideration.

The principal hypothesis posed by most authors of the aforementioned meta-analyses and a critical review on esophageal cancer epidemiology [37] is that H. pylori infection, with concomitant atrophy of the gastric corpus and loss of parietal cells, results in a reduction in reflux acidity and consequently in reflux esophagitis, Barrett's esophagus (BE), and EAC development.

There is evidence supporting the sequence GERD $\rightarrow \mathrm{BE} \rightarrow$ dysplasia $\rightarrow$ EAC and the implication of $H$. pylori separately in each single step to EAC, at least in certain subpopulations. BE is a complication of long-standing GERD and a well-known precursor lesion of EAC [38,39]; GERD plays an essential role in the pathophysiology and the clinical identification of $\mathrm{BE}$, which represents the only known complication derived from GERD [38,39]. The effect of $H$. pylori on BE varies according to geographic location. We showed that $H$. pylori infection is common in Greek patients with GERD, even in those without endoscopically proven reflux disease [40], and H. pylori eradication results in adequate control of GERD symptoms and improves esophagitis [41]. Consistent findings were reported by Schwizer et al [42], who also observed improvement in GERD symptoms after $H$. pylori treatment. Interestingly, other authors, previous supporters of the hypothesis that $H$. pylori "protects" against GERD, relented, claiming that $H$. pylori therapy does not cause or protect against GERD, and recommending $H$. pylori eradication in GERD [43]. Moreover, there are epidemiologic studies supporting our and others' data: a large-scale study (approximately 21,000 cases) reported that the decline in H.pylori infection parallels the reduction in peptic ulcer prevalence, and that the rise in GERD and/or reappearance of GERD following H. pylori therapy is rare. Contrary to expectations, patients 


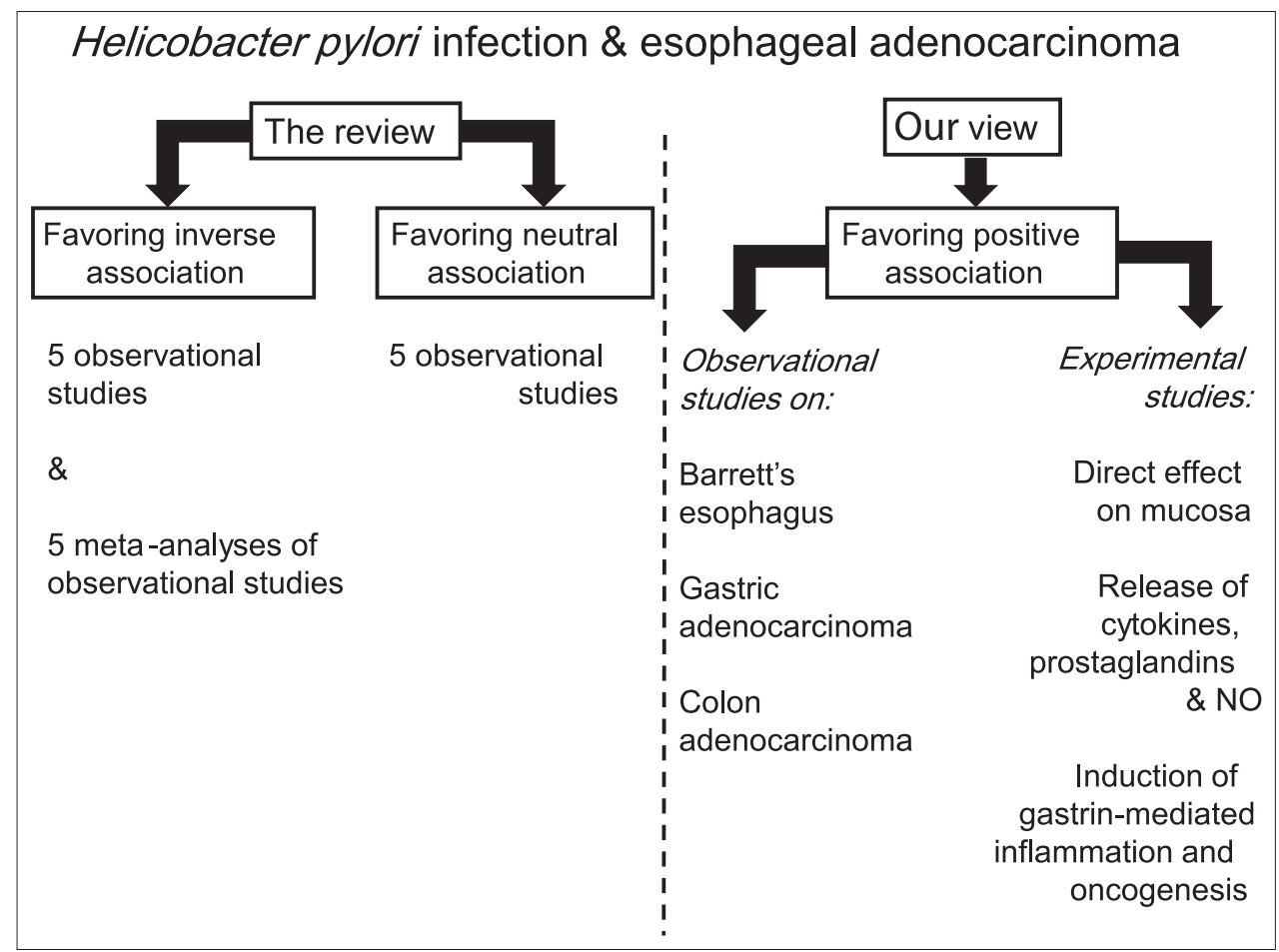

Figure 2 A synopsis of the review and our view. Five original studies and existing meta-analyses favor an inverse association between Helicobacter pylori infection and esophageal adenocarcinoma, whereas another five original studies favor a neutral association. On the other hand, our view is based on either clinical data linking H. pylori infection with Barrett's esophagus, and gastric and colon adenocarcinoma, or experimental studies showing a direct effect on esophageal mucosa or an indirect effect on inflammation and oncogenesis via mediators, including gastrin

hospitalized with duodenal ulcers (approximately 61,500 cases), apparently attributed to $H$. pylori infection, had a $70 \%$ increased risk of EAC [44]. Malaysians, who for a long time have had a low prevalence of $H$. pylori infection, also show a low incidence of GERD, BE and distal esophageal cancers, signifying that $H$. pylori infection is not protective against the abovementioned conditions and its absence may be beneficial [45]. The prevalence of EAC with persistent $H$. pylori infection is higher than that of EAC after eradication therapy [36,38]. Evidence further potentiates the consideration that $H$. pylori is not "protective" against anything, including GERD [36] and possibly its complications BE and EAC.

Apart from $H$. pylori, a number of other environmental agents (e.g., upper gastrointestinal microbiota) seem to play a role in GERD and BE pathogenesis; the presence of esophageal nitrate-reducing Campylobacter species in BE patients might suggest a connection with $\mathrm{BE}$ induction, maintenance, or exacerbation [41].

Beyond epidemiologic data, $H$. pylori might be involved in GERD pathophysiology via diverse mechanisms, such as: a) induction of mediators, cytokines and nitric oxide, which might disturb the lower esophageal sphincter (LES); b) direct injury of the esophageal mucosa by bacterial products; c) augmented release of prostaglandins that sensitizes afferent nerves and decreases LES pressure; and d) increased acidity due to gastrin induction that aggravates GERD [40].

At the molecular level, gastrin, caused by $H$. pylori infection, is an oncogenic growth agent that promotes upper and lower gastrointestinal tract oncogenesis. Specifically, gastrin appears to play an important role in neoplastic progression in BE. Gastrin stimulates proliferation via Janus Kinase (JAK)2 and Akt-dependent nuclear factor-kappa B (NF- $\kappa \mathrm{B})$ activation in Barrett's EAC cells, displays an anti-apoptotic effect via upregulation of Bcl-2 and survivin, and induces mitogenic and oncogenic cyclo-oxygenase (COX)-2 expression [38,39]. In this regard, $H$. pylori infection activates NF- $\kappa \mathrm{B}$, an oxidant-sensitive transcription regulator of inducible expression of inflammatory genes, including COX-2 that regulates gastrointestinal neoplasm cell growth and proliferation. Specifically, H. pylori infection promotes the expression of NF- $\mathrm{\kappa B}$ and COX-2 in esophageal epithelial cells, playing a role in the inflammatory process associated with BE and esophageal oncogenesis [38].

Upon colonizing the esophagus, $H$. pylori increases the severity of esophageal inflammation and the BE prevalence [38], as could be derived from the following data: a) H. pylori infection prevalence is high in $\mathrm{BE}$; b) neither $H$. pylori infection nor $H$. pylori infection by CagA positive strains decreases the risk of $\mathrm{BE}$ in some populations that have a high incidence of $H$. pylori infection; c) $H$. pylori infection might induce specific molecular changes (genetic instability, E-cadherin methylation, monoclonal antibody Das-1) linked with BE pathophysiology; and d) $H$. pylori promotes Ki-67 expression and greater Ki-67 esophageal expression was reported in BE patients compared with GERD controls. A progressive Ki-67 proliferation fraction was observed in the normal esophageal epithelium $\rightarrow \mathrm{BE} \rightarrow$ dysplasia $\rightarrow$ EAC sequence $[38,39]$. 
Insulin resistance (IR), the key MetS component [46], is connected with GERD, BE and EAC [4]. Since relative data indicate a relationship between $H$. pylori and IR [46] and other parameters of MetS [4], H. pylori-related MetS may contribute to the GERD $\rightarrow \mathrm{BE} \rightarrow$ EAC sequence in some ethnic populations [9]. Recent data show that lower serum adiponectin levels are associated with BE progression, while experimentally adiponectin induces an antitumor effect in Barrett's cell lines and prevents growth-factor signaling [4]. H. pylori therapy leads to an increase in levels of serum total adiponectin and its isoforms, thereby displaying a possible protective effect against malignant progression of BE [4].

Several studies support an association between $\mathrm{BE}$ and colonic neoplasms, including adenomas and adenocarcinomas [47-49]. It is conceivable that BE and colorectal neoplasms share a common, unidentified factor promoting the oncogenesis of BE-associatedEACand colorectal neoplasms. A potential association of both pathological conditions may be attributed to genetic predisposition or common environmental risk factors. H. pylori infection might promote both diseases [50]. Both $H$. pylori infection and BE are linked with an increased risk of the development of colorectal adenoma (CRA) and colorectal cancer (CRC) [12,50-52]. H. pylori infection appears to contribute to the GERD $\rightarrow$ $\mathrm{BE} \rightarrow \mathrm{EA}$ and $\mathrm{CRA} \rightarrow \mathrm{CRC}$ sequences, at least in certain populations, and its eradication may abrogate these oncogenic properties [12,51,52]. Specifically, active $H$. pylori infection appears to be involved in the pathogenesis of the normal colon epithelium $\rightarrow$ CRA $\rightarrow$ CRC sequence [12]. Excessive nicotinamide adenine dinucleotide phosphate (NADPH) oxidase activity and the production of reactive oxygen species (ROS) may promote oncogenic signaling, driving colorectal oncogenesis [13]. Likewise, in the $H$. pylori-related GERD $\rightarrow$ $\mathrm{BE} \rightarrow \mathrm{EAC}$ sequence, NADPH activation and NADPH-derived ROS may cause DNA damage, thereby contributing to the progression from $\mathrm{BE}$ to EAC [13].

In conclusion, existing epidemiologic studies provided inconclusive data on an inverse or a neutral association between $H$. pylori infection and EAC, whereas metaanalyses of observational studies favor an inverse association. A particular drawback of most original studies is confounding factors, i.e., multiple factors that were not taken into consideration in the study design or the analysis of data, but may possibly contribute to the pathogenesis of EAC. This might have affected the results of the metaanalyses, since they included original studies that did not adequately adjust for potential confounders. Furthermore, the source of heterogeneity, when it was observed, was not evaluated in the meta-analyses. In this regard, well-designed prospective cohort studies with a powered sample size are required, in which potential confounders should be taken into account. This may resolve the paradox of the positive association of $H$. pylori infection with GERD or BE, but not with EAC, as well as the paradox of the oncogenic effect of H. pylori infection on gastric cancer and CRC, but not on EAC. Metabolomics may also prove helpful in this direction in the near future, as the $H$. pylori-related metabolites may provide further data.

\section{References}

1. Kao CY, Sheu BS, Wu JJ. Helicobacter pylori infection: An overview of bacterial virulence factors and pathogenesis. Biomed $J$ 2016;39:14-23.

2. Lanas A, Chan FKL. Peptic ulcer disease. Lancet 2017;390:613-624.

3. Schistosomes, liver flukes and Helicobacter pylori. IARC Working Group on the Evaluation of Carcinogenic Risks to Humans. Lyon, 7-14 June 1994. IARC Monogr Eval Carcinog Risks Hum 1994;61:1-241.

4. Franceschi F, Gasbarrini A, Polyzos SA, Kountouras J. Extragastric diseases and Helicobacter pylori. Helicobacter 2015;20(Suppl 1):40-46.

5. Kountouras J, Polyzos SA, Katsinelos P, et al. Cardio-cerebrovascular disease and Helicobacter pylori-related metabolic syndrome: We consider eradication therapy as a potential cardio-cerebrovascular prevention strategy. Int J Cardiol 2017;229:17-18.

6. Wang XL, Zeng J, Yang Y, et al. Helicobacter pylori filtrate induces Alzheimer-like tau hyperphosphorylation by activating glycogen synthase kinase-3 $\beta$. J Alzheimers Dis 2015;43:153-165.

7. Deretzi G, Gavalas E, Boziki M, et al. Impact of Helicobacter pylori on multiple sclerosis-related clinically isolated syndrome. Acta Neurol Scand 2016;133:268-275.

8. Gavalas E, Kountouras J, Boziki M, et al. Relationship between Helicobacter pylori infection and multiple sclerosis. Ann Gastroenterol 2015;28:353-356.

9. Kountouras J, Polyzos SA, Zeglinas C, et al. Helicobacter pylori-related metabolic syndrome as predictor of progression to esophageal carcinoma in a subpopulation-based Barrett's esophagus cohort. Gastrointest Endosc 2017;85:462-463.

10. Raderer M, Wrba F, Kornek G, et al. Association between Helicobacter pylori infection and pancreatic cancer. Oncology 1998;55:16-19.

11. Shmuely H, Passaro D, Figer A, et al. Relationship between Helicobacter pylori CagA status and colorectal cancer. Am J Gastroenterol 2001;96:3406-3410.

12. Kountouras J, Kapetanakis N, Zavos C, Polyzos SA, Romiopoulos I. Active Helicobacter pylori infection on colorectal mucosa-adenomatous polyp-adenocarcinoma sequence. Eur J Gastroenterol Hepatol 2014;26:243-244.

13. Kountouras J, Boziki M, Polyzos SA, et al. Impact of reactive oxygen species generation on Helicobacter pylori-related extragastric diseases: a hypothesis. Free Radic Res 2017;51:73-79.

14. Wijetunge S, Ma Y, DeMeester S, Hagen J, DeMeester T, Chandrasoma P. Association of adenocarcinomas of the distal esophagus, "gastroesophageal junction," and "gastric cardia" with gastric pathology. Am J Surg Pathol 2010;34:1521-1527.

15. Uemura N, Kondo T. Current status of proteomics of esophageal carcinoma. Expert Rev Proteomics 2016 Oct 8:1-12. [Epub ahead of print] doi: 10.1080/14789450.2016.12424181-12

16. Napier KJ, Scheerer M, Misra S. Esophageal cancer: a review of epidemiology, pathogenesis, staging workup and treatment modalities. World J Gastrointest Oncol 2014;6:112-120.

17. Short MW, Burgers KG, Fry VT. Esophageal cancer. Am Fam Physician 2017;95:22-28.

18. Slim K, Nini E, Forestier D, Kwiatkowski F, Panis Y, Chipponi J. Methodological index for non-randomized studies (minors): development and validation of a new instrument. ANZ J Surg 2003;73:712-716.

19. Abraham NS, Byrne CJ, Young JM, Solomon MJ. Meta-analysis of well-designed nonrandomized comparative studies of surgical procedures is as good as randomized controlled trials. J Clin Epidemiol 2010;63:238-245.

20. Papaconstantinou I, Zeglinas C, Gazouli M, et al. The impact of peri-operative anti-TNF treatment on anastomosis-related 
complications in Crohn's disease patients. A critical review. J Gastrointest Surg 2014;18:1216-1224.

21. Whiteman DC, Parmar P, Fahey P, et al; Australian Cancer Study. Association of Helicobacter pylori infection with reduced risk for esophageal cancer is independent of environmental and genetic modifiers. Gastroenterology 2010;139:73-83.

22. Früh M, Zhou W, Zhai R, et al. Polymorphisms of inflammatory and metalloproteinase genes, Helicobacter pylori infection and the risk of oesophageal adenocarcinoma. Br J Cancer 2008;98:689-692.

23. Anderson LA, Murphy SJ, Johnston BT, et al. Relationship between Helicobacter pylori infection and gastric atrophy and the stages of the oesophageal inflammation, metaplasia, adenocarcinoma sequence: results from the FINBAR case-control study. Gut 2008;57:734-739.

24. de Martel C, Llosa AE, Farr SM, et al. Helicobacter pylori infection and the risk of development of esophageal adenocarcinoma. J Infect Dis 2005;191:761-767.

25. Ye W, Held M, Lagergren J, et al. Helicobacter pylori infection and gastric atrophy: risk of adenocarcinoma and squamous-cell carcinoma of the esophagus and adenocarcinoma of the gastric cardia. J Natl Cancer Inst 2004;96:388-396.

26. Simán JH, Engstrand L, Berglund G, Forsgren A, Florén $\mathrm{CH}$. Helicobacter pylori and CagA seropositivity and its association with gastric and oesophageal carcinoma. Scand J Gastroenterol 2007;42:933-940.

27. Wu AH, Crabtree JE, Bernstein L, et al. Role of Helicobacter pylori CagA+ strains and risk of adenocarcinoma of the stomach and esophagus. Int J Cancer 2003;103:815-821.

28. El-Omar EM, Rabkin CS, Gammon MD, et al. Increased risk of noncardia gastric cancer associated with proinflammatory cytokine gene polymorphisms. Gastroenterology 2003;124:1193-1201.

29. Vieth M, Masoud B, Meining A, Stolte M. Helicobacter pylori infection: protection against Barrett's mucosa and neoplasia? Digestion 2000;62:225-231.

30. Peek RM Jr, Vaezi MF, Falk GW, et al. Role of Helicobacter pylori cagA $(+)$ strains and specific host immune responses on the development of premalignant and malignant lesions in the gastric cardia. Int J Cancer 1999;82:520-524.

31. Rokkas T, Pistiolas D, Sechopoulos P, Robotis I, Margantinis G. Relationship between Helicobacter pylori infection and esophageal neoplasia: a meta-analysis. Clin Gastroenterol Hepatol 2007;5:1413-1417.

32. Zhuo X, Zhang Y, Wang Y, Zhuo W, Zhu Y, Zhang X. Helicobacter pylori infection and oesophageal cancer risk: association studies via evidence-based meta-analyses. Clin Oncol ( $R$ Coll Radiol) 2008;20:757-762.

33. Islami F, Kamangar F. Helicobacter pylori and esophageal cancer risk: a meta-analysis. Cancer Prev Res (Phila) 2008;1:329-338.

34. Xie FJ, Zhang YP, Zheng QQ, et al. Helicobacter pylori infection and esophageal cancer risk: an updated meta-analysis. World $J$ Gastroenterol 2013;19:6098-6107.

35. Nie S, Chen T, Yang X, Huai P, Lu M. Association of Helicobacter pylori infection with esophageal adenocarcinoma and squamous cell carcinoma: a meta-analysis. Dis Esophagus 2014;27:645-653.

36. Graham DY. Helicobacter pylori is not and never was "protective" against anything, including GERD. Dig Dis Sci 2003;48:629-630.

37. Thrift AP. The epidemic of oesophageal carcinoma: Where are we now? Cancer Epidemiol 2016;41:88-95.

38. Kountouras J, Chatzopoulos D, Zavos C, et al. Helicobacter pylori infection might contribute to esophageal adenocarcinoma progress in subpopulations with gastroesophageal reflux disease and Barrett's esophagus. Helicobacter 2012;17:402-403.

39. Kountouras J, Chatzopoulos D, Zavos C. Eradication of Helicobacter pylori might halt the progress to oesophageal adenocarcinoma in patients with gastro-oesophageal reflux disease and Barrett's oesophagus. Med Hypotheses 2007;68:1174-1175.

40. Kountouras J, Zavos C, Chatzopoulos D, Katsinelos P. Helicobacter pylori and gastro-oesophageal reflux disease. Lancet 2006;368:986.

41. Kountouras J, Zavos C, Polyzos SA, Katsinelos P. Helicobacter pylori infection and gastroesophageal reflux disease - Barrett's esophagus sequence "dilemma". Ann Gastroenterol 2015;28:153.

42. Schwizer W, Thumshirn M, Dent J, et al. Helicobacter pylori and symptomatic relapse of gastro-oesophageal reflux disease: a randomised controlled trial. Lancet 2001;357:1738-1742.

43. Moayyedi P. Should we test for Helicobacter pylori before treating gastroesophageal reflux disease? Can J Gastroenterol 2005; 19:425-427.

44. Bahmanyar S, Zendehdel K, Nyrén O, Ye W. Risk of oesophageal cancer by histology among patients hospitalised for gastroduodenal ulcers. Gut 2007;56:464-468.

45. Lee YY, Mahendra Raj S, Graham DY. Helicobacter pylori infection-a boon or a bane: lessons from studies in a lowprevalence population. Helicobacter 2013;18:338-346.

46. Polyzos SA, Kountouras J, Zavos C, Deretzi G. The association between Helicobacter pylori infection and insulin resistance: a systematic review. Helicobacter 2011;16:79-88.

47. Siersema PD, Yu S, Sahbaie P, et al. Colorectal neoplasia in veterans is associated with Barrett's esophagus but not with proton-pump inhibitor or aspirin/NSAID use. Gastrointest Endosc 2006;63:581-586.

48. Andrici J, Tio M, Cox MR, Eslick GD. Meta-analysis: Barrett's oesophagus and the risk of colonic tumours. Aliment Pharmacol Ther 2013;37:401-410.

49. Sonnenberg A, Genta RM. Barrett's metaplasia and colonic neoplasms: a significant association in a 203,534-patient study. Dig Dis Sci 2013;58:2046-2051.

50. Kountouras J, Zavos C, Chatzopoulos D. Pathogenetic links between colorectal neoplasia and Barrett's esophagus. Gastrointest Endosc 2006;64:298.

51. Kountouras J, Zavos C, Chatzopoulos D, et. Letter: is Helicobacter pylori behind Barrett's oesophagus and colorectal neoplasms? Aliment Pharmacol Ther 2013;37:837.

52. Kapetanakis N, Kountouras J, Zavos C, et al. Association of Helicobacter pylori infection with colorectal cancer. Immunogastroenterology 2013;2:47-56. 